\title{
On certain subclasses of multivalent functions defined by multiplier transformations
}

\author{
Jagannath Patel ${ }^{1}$, Nak Eun Cho $^{2^{*}}$ and Ashis Kumar Palit ${ }^{3}$ \\ Dedicated to Professor Hari M Srivastava
}

"Correspondence:

necho@pknu.ac.kr

${ }^{2}$ Department of Applied

Mathematics, Pukyong National University, Busan, Korea

Full list of author information is

available at the end of the article

\begin{abstract}
The purpose of the present paper is to introduce and investigate various properties of a certain class of multivalent functions in the open unit disk defined by a multiplier transformation. In particular, we obtain some inclusion relationships and integral preserving properties of this class of functions. Relevant connections of the results presented in this paper with various known results are also pointed out.

MSC: $30 C 45$
\end{abstract}

Keywords: analytic function; multivalent function; multiplier transformation; subordination; integral operator

\section{Introduction}

Let $\mathcal{A}_{p}$ denote the class of functions of the form

$$
f(z)=z^{p}+\sum_{k=1}^{\infty} a_{p+k} z^{p+k}
$$

which are analytic in the open unit disk $\mathbb{U}=\{z \in \mathbb{C}:|z|<1\}$. We write $\mathcal{A}_{1}=\mathcal{A}$.

Suppose that $f$ and $g$ are analytic in $\mathbb{U}$. We say that the function $f$ is subordinate to $g$ in $\mathbb{U}$, and we write $f \prec g$ or $f(z) \prec g(z)(z \in \mathbb{U})$, if there exists an analytic function $\omega$ in $\mathbb{U}$ with $\omega(0)=0$ and $|\omega(z)|<1$ for all $z \in \mathbb{U}$ such that $f(z)=g(\omega(z))$ in $\mathbb{U}$. If $g$ is univalent in $\mathbb{U}$, then the following equivalence relationship holds true:

$$
f(z) \prec g(z) \quad \Longleftrightarrow \quad f(0)=g(0) \quad \text { and } \quad f(\mathbb{U}) \subset g(\mathbb{U}) \text {. }
$$

For functions $f$ given by (1.1) and $g(z)=z^{p}+\sum_{k=1}^{\infty} b_{p+k} z^{p+k}$, the Hadamard product (or convolution) of $f$ and $g$ is defined by

$$
(f * g)(z)=f(z) * g(z)=z^{p}+\sum_{k=1}^{\infty} a_{p+k} b_{p+k} z^{p+k} .
$$

For fixed parameters $A, B(-1 \leqq B<A \leqq 1)$, let $\mathcal{P}(A, B)$ be the class of functions of the form

$$
\varphi(z)=1+c_{1} z+c_{2} z^{2}+\cdots
$$

@ 2013 Patel et al.; licensee Springer. This is an Open Access article distributed under the terms of the Creative Commons Attribution License (http://creativecommons.org/licenses/by/2.0), which permits unrestricted use, distribution, and reproduction in any medium, provided the original work is properly cited. 
which are analytic in $\mathbb{U}$ and satisfy the condition

$$
\varphi(z) \prec \frac{1+A z}{1+B z} \quad(z \in \mathbb{U}) .
$$

The class $\mathcal{P}(A, B)$ was investigated in [1]. We denote by $\mathcal{S}_{p}^{*}(A, B)$ the class of functions $f \in \mathcal{A}_{p}$ such that $z f^{\prime} / p f \in \mathcal{P}(A, B)$. Analogously, $\mathcal{K}(A, B)$ is the class of functions $f \in \mathcal{A}_{p}$ such that $\left(z f^{\prime}\right)^{\prime} \mid p f^{\prime} \in \mathcal{P}(A, B)$. It is easily seen that

$$
\mathcal{S}_{p}^{*}\left(1-\frac{2 \rho}{p},-1\right)=\mathcal{S}_{p}^{*}(\rho) \quad \text { and } \quad \mathcal{K}_{p}\left(1-\frac{2 \rho}{p},-1\right)=\mathcal{K}_{p}(\rho) \quad(0 \leqq \rho<p)
$$

the subclasses of $\mathcal{A}_{p}$, which are respectively, $p$-valently starlike of order $\rho$ and $p$-valently convex of order $\rho$ in $\mathbb{U}$. We also note that

$$
\mathcal{S}_{p}^{*}(\rho) \subseteq \mathcal{S}_{p}^{*}(0)=\mathcal{S}_{p}^{*} \quad \text { and } \quad \mathcal{K}_{p}(\rho) \subseteq \mathcal{K}_{p}(0)=\mathcal{K}_{p} \quad(0 \leqq \rho<p),
$$

where $\mathcal{S}_{p}^{*}$ and $\mathcal{K}_{p}$ are the subclasses of $\mathcal{A}_{p}$ consisting of functions that are $p$-valently starlike and $p$-valently convex in $\mathbb{U}$, respectively.

In the present investigation, we shall make use of the Gauss hypergeometric function ${ }_{2} F_{1}$ defined in $\mathbb{U}$ by

$$
{ }_{2} F_{1}(a, b ; c ; z)=\sum_{k=0}^{\infty} \frac{(a)_{k}(b)_{k}}{(c)_{k}} \frac{z^{k}}{k !} \quad\left(a, b, c \in \mathbb{C} ; c \notin \mathbb{Z}_{0}^{-}=\{0,-1,-2, \ldots\}\right),
$$

where $(x)_{n}$ denotes the Pochhammer symbol (or shifted factorial) given by

$$
(x)_{n}= \begin{cases}x(x+1)(x+2) \cdots(x+n-1) & (n \in \mathbb{N}) \\ 1 & (n=0)\end{cases}
$$

We note that the series defined by (1.3) converges absolutely for all $z \in \mathbb{U}$ and hence represents an analytic function in $\mathbb{U}[2$, Chapter 14].

Motivated by the multiplier transformation introduced in [3] on $\mathcal{A}$, we introduce an operator $\phi_{p}(n, \lambda)$ on $\mathcal{A}_{p}$ by

$$
\phi_{p}(n, \lambda)(z)=z^{p}+\sum_{k=1}^{\infty}\left(\frac{p+k+\lambda}{p+\lambda}\right) z^{p+k} \quad(\lambda>-p, n \in \mathbb{Z}=\{0, \pm 1, \pm 2, \ldots\} ; z \in \mathbb{U}) .
$$

The operator $\phi_{p}(n, \lambda)$ is related to the multiplier transformation studied in [4].

Corresponding to the function $\phi_{p}(n, \lambda)$, we define a new function $\phi_{p}^{(\dagger)}(n, \lambda)$ in terms of the Hadamard product by

$$
\phi_{p}(n, \lambda)(z) * \phi_{p}^{(\dagger)}(n, \lambda)(z)=\frac{z^{p}}{(1-z)^{p+\mu}} \quad(\mu>-p ; z \in \mathbb{U}) .
$$

We now introduce the operator $I_{p}^{n}(\lambda, \mu): \mathcal{A}_{p} \longrightarrow \mathcal{A}_{p}$ by

$$
I_{p}^{n}(\lambda, \mu) f(z)=\phi_{p}^{(\dagger)}(n, \lambda)(z) * f(z) \quad(n \in \mathbb{Z} ; \lambda, \mu>-p) .
$$


If the function $f$ is given by (1.1), then from (1.4) and (1.5) we deduce that

$$
I_{p}^{n}(\lambda, \mu) f(z)=z^{p}+\sum_{k=1}^{\infty} \frac{(p+\mu)_{k}}{(1)_{k}}\left(\frac{p+\lambda}{p+k+\lambda}\right)^{n} a_{p+k} z^{p+k} \quad(z \in \mathbb{U}) .
$$

In view of (1.5), it follows that

$$
z\left(I_{p}^{n}(\lambda, \mu) f\right)^{\prime}(z)=(p+\lambda) I_{p}^{n-1}(\lambda, \mu) f(z)-\lambda I_{p}^{n}(\lambda, \mu) f(z) \quad\left(f \in \mathcal{A}_{p} ; z \in \mathbb{U}\right) .
$$

In particular, we note that for $z \in \mathbb{U}$,

$$
\begin{aligned}
& I_{p}^{0}(0,1-p) f(z)=f(z) \\
& I_{p}^{1}(\delta, 1-p) f(z)=\left((p+\delta) \int_{0}^{z} t^{\delta-1} f(t) d t\right) / z^{\delta} \quad(\delta>-p)[c f . \text { Eqn. }(3.10)] \\
& I_{p}^{-1}(\lambda, 1-p) f(z)=\left(z f^{\prime}(z)+\lambda f(z)\right) /(p+\lambda) \\
& I_{p}^{-2}(0,1-p) f(z)=\left(z^{2} f^{\prime \prime}(z)+z f^{\prime}(z)\right) / p^{2} \quad \text { and } \\
& I_{p}^{-3}(0,1-p) f(z)=\left(z^{3} f^{\prime \prime \prime}(z)+3 z^{2} f^{\prime \prime}(z)+z f^{\prime}(z)\right) / p^{3}
\end{aligned}
$$

The operator $I_{p}^{n}(\lambda, 1-p)\left(n \in \mathbb{Z}_{0}^{-}\right)$is closely related to the Sálágean derivative operator [5]. The operator $I_{\lambda}^{n}=I_{1}^{n}(\lambda, 0)$ was recently studied in $[3,6,7]$. For any $n \in \mathbb{Z}$, the operator $I_{n}=I_{1}^{n}(1,0)$ was studied in $[8]$.

By using the operator $I_{p}^{n}(\lambda, \mu)$, we introduce the subclass of $\mathcal{A}_{p}$ as follows.

Definition For fixed parameters $A, B(-1 \leqq B<A \leqq 1), n \in \mathbb{Z}, \lambda, \mu>-p$ and $\alpha \geqq 0$, we say that a function $f \in \mathcal{A}_{p}$ is in the class $\mathcal{S}_{p, \lambda, \mu}^{n}(\alpha ; A, B)$ if

$$
(1-\alpha) \frac{I_{p}^{n}(\lambda, \mu) f(z)}{I_{p}^{n+1}(\lambda, \mu) f(z)}+\alpha \frac{I_{p}^{n-1}(\lambda, \mu) f(z)}{I_{p}^{n}(\lambda, \mu) f(z)} \prec \frac{1+A z}{1+B z} \quad(z \in \mathbb{U}) .
$$

It is readily seen that

$$
\mathcal{S}_{p, 0,1-p}^{0}(1 ; A, B)=\mathcal{S}_{p}^{*}(A, B) \quad \text { and } \quad \mathcal{S}_{p, 0,1-p}^{-1}(1 ; A, B)=\mathcal{K}_{p}(A, B)
$$

For the sake of convenience, we write

$$
\mathcal{S}_{p, \lambda, \mu}^{n}(A, B)=\mathcal{S}_{p, \lambda, \mu}^{n}(1 ; A, B)=\left\{f \in \mathcal{A}_{p}: \frac{I_{p}^{n}(\lambda, \mu) f(z)}{I_{p}^{n+1}(\lambda, \mu) f(z)} \prec \frac{1+A z}{1+B z}, z \in \mathbb{U}\right\}
$$

The object of the present paper is to investigate some inclusion properties of the class $\mathcal{S}_{p, \lambda, \mu}^{n}(\alpha ; A, B)$. Integral-preserving and convolution properties in connection with the operator $I_{p}^{n}(\lambda, \mu)$ are also considered. Relevant connections of the results presented here with those obtained in the earlier works are pointed out.

\section{Preliminaries}

We denote by $\mathcal{H}$ the class of all analytic functions in $\mathbb{U}$ and by $\mathcal{B}$ the class of functions $\Omega \in \mathcal{H}$ such that $\omega(0)=0$ and $|\Omega(z)|<1$ for $z \in \mathbb{U}$.

We shall need the following lemmas to prove our results. 
Lemma 1 ([9], see also [10, p.71]) Let $h$ be analytic and convex (univalent) in $\mathbb{U}$ with $h(0)=1$. Suppose also that the function $\varphi$ defined by (1.2) is analytic in $\mathbb{U}$. If

$$
\varphi(z)+\frac{z \varphi^{\prime}(z)}{\kappa} \prec h(z) \quad(\kappa \neq 0, \Re(\kappa) \geqq 0 ; z \in \mathbb{U}),
$$

then

$$
\varphi(z) \prec \psi(z)=\frac{\kappa}{z^{\kappa}} \int_{0}^{z} t^{\kappa-1} h(t) d t \prec h(z) \quad(z \in \mathbb{U})
$$

and $\psi$ is the best dominant of (2.1).

Lemma $2\left[10\right.$, p.35] Suppose that the function $\Psi: \mathbb{C}^{2} \times \mathbb{U} \longrightarrow \mathbb{C}$ satisfies the condition

$$
\Psi(i x, y ; z) \leqq \varepsilon
$$

for $\varepsilon>0$, real $x, y \leqq-\left(1+x^{2}\right) / 2$ and all $z \in \mathbb{U}$. If the function $\varphi$, given by (1.2) is analytic in $\mathbb{U}$ and

$$
\operatorname{Re}\left(\Psi\left(\varphi(z), z \varphi^{\prime}(z) ; z\right)\right)>\varepsilon
$$

then $\operatorname{Re}(\varphi(z))>0$ in $\mathbb{U}$.

Lemma 3 [11] Let $0<\gamma_{1}<\gamma<1$ and $\Theta \in \mathcal{H}$ satisfy

$$
\Theta(z) \prec 1+\gamma_{1} z, \quad \Theta(0)=1 .
$$

(i) If $\varphi \in \mathcal{H}, \varphi(0)=1$ and satisfies

$$
\Theta(z)(\beta+(1-\beta) \varphi(z)) \prec 1+\gamma z \quad(z \in \mathbb{U})
$$

where

$$
\beta= \begin{cases}\frac{1-\gamma}{1+\gamma_{1}} & \left(0<\gamma_{1}+\gamma \leqq 1\right) \\ \frac{1-\left(\gamma_{1}^{2}+\gamma^{2}\right)}{2\left(1-\gamma_{1}^{2}\right)} & \left(\gamma_{1}^{2}+\gamma^{2} \leqq 1 \leqq \gamma_{1}+\gamma\right)\end{cases}
$$

then $\operatorname{Re}(\varphi(z))>0$ in $\mathbb{U}$.

(ii) If $\omega \in \mathcal{H}$ with $\omega(0)=0$ satisfies

$$
\Theta(z)(1+\omega(z)) \prec 1+\gamma z \quad(z \in \mathbb{U}),
$$

then, for $0<2 \gamma_{1}+\gamma \leqq 1$, we have

$$
|\omega(z)| \leqq \frac{\gamma_{1}+\gamma}{1-\gamma_{1}} \quad(z \in \mathbb{U}) .
$$

The value of $\beta$ in (2.2) and the bound in (2.3) are best possible. 
Lemma 4 [11] If $\omega \in \mathcal{B}$ and

$$
\varphi(z)=\frac{1+\gamma \omega(z)}{1+\gamma \delta^{\prime} \int_{0}^{1} t^{\delta^{\prime}-1} \omega(t z) d t} \quad\left(0<\gamma<1, \delta^{\prime}>0, z \in \mathbb{U}\right)
$$

then $\operatorname{Re}(\varphi(z))>\beta(0 \leqq \beta<1)$ in $\mathbb{U}$, where

$$
\beta= \begin{cases}\frac{1-\gamma}{1+\gamma \delta_{1}} & \left(0<\gamma \leqq \frac{1}{1+\delta_{1}}\right) \\ \frac{1-\gamma^{2}\left(1+\delta_{1}^{2}\right)}{2\left(1-\gamma^{2} \delta_{1}^{2}\right)} & \left(\frac{1}{1+\delta_{1}} \leqq \gamma \leqq \frac{1}{\sqrt{1+\delta_{1}^{2}}}\right)\end{cases}
$$

and $\delta_{1}=\delta^{\prime} /\left(1+\delta^{\prime}\right)$. Further, for $0<\gamma \leqq 1 /\left(1+2 \delta_{1}\right)$, we have

$$
|\varphi(z)-1| \leqq \frac{\gamma\left(1+\delta_{1}\right)}{1-\gamma \delta_{1}} \quad(z \in \mathbb{U})
$$

The value of $\beta$ in (2.4) and the bound in (2.5) are best possible.

3 Inclusion relationships for the class $\mathcal{S}_{p, \lambda, \mu}^{n}(\alpha ; A, B)$

Unless otherwise mentioned, we assume throughout the sequel that

$$
n \in \mathbb{Z}, \quad \lambda, \mu>-p, \quad \alpha>0 \quad \text { and } \quad-1 \leqq B<A \leqq 1 .
$$

Theorem 1 We have

$$
\mathcal{S}_{p, \lambda, \mu}^{n}(\alpha ; A, B) \subset \mathcal{S}_{p, \lambda, \mu}^{n}(A, B) .
$$

Further, for $f \in \mathcal{S}_{p, \lambda, \mu}^{n}(\alpha ; A, B)$, we also have

$$
\frac{I_{p}^{n}(\lambda, \mu) f(z)}{I_{p}^{n+1}(\lambda, \mu) f(z)} \prec q(z)=\frac{\alpha}{(p+\lambda) Q(z)} \quad(z \in \mathbb{U})
$$

where

$$
Q(z)= \begin{cases}\int_{0}^{1} t^{\frac{p+\lambda}{\alpha}-1}\left(\frac{1+t B z}{1+B z}\right)^{\frac{(p+\lambda)(B-A)}{\alpha B}} d t & (B \neq 0), \\ \int_{0}^{1} t^{\frac{p+\lambda}{\alpha}-1} \exp \left(\frac{(p+\lambda)(t-1) A}{\alpha} z\right) d t & (B=0)\end{cases}
$$

and $q$ is the best dominant of (3.1). Moreover, if $A \leqq-\frac{\alpha B}{p+\lambda}(-1 \leqq B<0)$, then

$$
\mathcal{S}_{p, \lambda, \mu}^{n}(\alpha ; A, B) \subset \mathcal{S}_{p, \lambda, \mu}^{n}(1-2 \rho,-1),
$$

where $\rho=\left[{ }_{2} F_{1}\left(1, \frac{(p+\lambda)(B-A)}{\alpha B}, \frac{p+\lambda}{\alpha}+1 ; \frac{B}{B-1}\right)\right]^{-1}$. The bound $\rho$ is best possible.

Proof Let $f \in \mathcal{S}_{p, \lambda, \mu}^{n}(\alpha ; A, B)$. Suppose that

$$
g(z)=z\left(\frac{I_{p}^{n+1}(\lambda, \mu) f(z)}{z^{p}}\right)^{1 /(p+\lambda)}
$$


and $r_{1}=\sup \{r: g(z) \neq 0,0<|z|<r<1\}$. Choosing the principal branch of $g$, we note that $g$ is single-valued and analytic in $\mathbb{U}_{r_{1}}=\left\{z:|z|<r_{1}\right\}$. Taking the logarithmic differentiation in (3.4) and using identity (1.6) in the resulting equation, we get that

$$
\varphi(z)=\frac{z g^{\prime}(z)}{g(z)}=\frac{I_{p}^{n}(\lambda, \mu) f(z)}{I_{p}^{n+1}(\lambda, \mu) f(z)}
$$

is of the form (1.2) and is analytic in $\mathbb{U}_{r_{1}}$. Again, carrying out logarithmic differentiation in (3.5) and using (1.6), we deduce that

$$
(1-\alpha) \frac{I_{p}^{n}(\lambda, \mu) f(z)}{I_{p}^{n+1}(\lambda, \mu) f(z)}+\alpha \frac{I_{p}^{n-1}(\lambda, \mu) f(z)}{I_{p}^{n}(\lambda, \mu) f(z)}=\varphi(z)+\frac{\alpha}{p+\lambda} \frac{z \varphi^{\prime}(z)}{\varphi(z)} \prec \frac{1+A z}{1+B z} \quad\left(z \in \mathbb{U}_{r_{1}}\right) .
$$

Hence, by applying the result [12, Corollary 3.2], we obtain

$$
\varphi(z) \prec q(z)=\frac{\alpha}{(p+\lambda) Q(z)} \prec \frac{1+A z}{1+B z} \quad\left(z \in \mathbb{U}_{r_{1}}\right),
$$

where $q$ is the best dominant of (3.1) and $Q$ is given by (3.2).

The proof of the remaining part can now be deduced along the same lines as in [13, Theorem 1]. The bound $\rho$ in (3.3) is best possible as $q$ is the best dominant of (3.1). This evidently completes the proof of the theorem.

Setting $n=-1, \lambda=0$ and $\mu=1-p$ in Theorem 1 , we get the following corollary.

Corollary 1 If $A \leqq-\alpha B / p(-1 \leqq B<0)$ and $f \in \mathcal{A}_{p}$ satisfies

$$
(1-\alpha) \frac{z f^{\prime}(z)}{f(z)}+\alpha\left(1+\frac{z f^{\prime \prime}(z)}{f^{\prime}(z)}\right) \prec \frac{p(1+A z)}{1+B z} \quad(z \in \mathbb{U}),
$$

then

$$
\operatorname{Re}\left(\frac{z f^{\prime}(z)}{f(z)}\right)>p\left[{ }_{2} F_{1}\left(1, \frac{p(B-A)}{\alpha B}, \frac{p}{\alpha}+1 ; \frac{B}{B-1}\right)\right]^{-1} \quad(z \in \mathbb{U}) .
$$

The result is best possible.

In the special case when $n=0, \mu=1-p, A=1-(2(\eta+\lambda \alpha) /(p+\lambda))(0 \leqq \eta<1)$ and $B=-1$, Theorem 1 gives the following.

Corollary 2 If $\max \{-\lambda \alpha,(p+\lambda-2 \lambda \alpha-\alpha) / 2\}<\eta<p+\lambda-\lambda \alpha$ and $f \in \mathcal{A}_{p}$ satisfies

$$
\operatorname{Re}\left((1-\alpha) \frac{z^{\lambda} f(z)}{\int_{0}^{z} t^{\lambda-1} f(t) d t}+\alpha \frac{z f^{\prime}(z)}{f(z)}\right)>\eta \quad(z \in \mathbb{U})
$$

then

$$
\operatorname{Re}\left(\frac{z^{\lambda} f(z)}{\int_{0}^{z} t^{\lambda-1} f(t) d t}\right)>(p+\lambda)\left[{ }_{2} F_{1}\left(1, \frac{2(p+\lambda-\lambda \alpha-\eta)}{\alpha}, \frac{p+\lambda}{\alpha}+1 ; \frac{1}{2}\right)\right]^{-1} \quad(z \in \mathbb{U}) .
$$

The result is best possible. 
Remarks 1. Putting $A=1$ and $B=-1$ in Corollary 1 , we find that for $\alpha \geqq p$ and $z \in \mathbb{U}$,

$$
\operatorname{Re}\left((1-\alpha) \frac{z f^{\prime}(z)}{f(z)}+\alpha\left(1+\frac{z f^{\prime \prime}(z)}{f^{\prime}(z)}\right)\right)>0 \Longrightarrow f \in \mathcal{S}_{p}^{*}\left(\frac{p \Gamma(1+(2 p / \alpha))}{\sqrt{\pi} \Gamma(1+(p / \alpha))}\right),
$$

which in turn implies that

$$
f \in \mathcal{K}_{p}\left(\frac{p(\alpha-1) \Gamma(1+(2 p / \alpha))}{\sqrt{\pi} \alpha \Gamma(1+(p / \alpha))}\right) .
$$

For $p=1$, this result is contained in [14].

2. Setting $\alpha=1, A=1-(2 \eta / p)(0 \leqq \eta<p)$ and $B=-1$ in Corollary 1 and $\alpha=1$ in Corollary 2 , we get the corresponding result obtained in [15].

Theorem 2 For $0 \leqq \eta<p$, we have

$$
f \in \mathcal{S}_{p, \lambda, \mu}^{n}\left(0 ; 1-\frac{2 \eta}{p},-1\right) \Longrightarrow f \in \mathcal{S}_{p, \lambda, \mu}^{n}\left(\alpha ; 1-\frac{2 \eta}{p},-1\right) \quad(|z|<R)
$$

where

$$
R= \begin{cases}\frac{p \alpha+(p+\lambda)(p-\eta)-\sqrt{(p \alpha+(p+\lambda)(p-\eta))^{2}-p(p+\lambda)^{2}(p-2 \eta)}}{(p+\lambda)(p-2 \eta)} & \left(\eta \neq \frac{p}{2}\right), \\ \frac{p+\lambda}{p+\lambda+2 \alpha} & \left(\eta=\frac{p}{2}\right) .\end{cases}
$$

The bound $R$ is best possible.

Proof Setting

$$
\frac{I_{p}^{n}(\lambda, \mu) f(z)}{I_{p}^{n+1}(\lambda, \mu) f(z)}=\frac{\eta}{p}+\left(1-\frac{\eta}{p}\right) u(z) \quad(z \in \mathbb{U}),
$$

we see that $u$ is of the form (1.2), analytic and has a positive real part in $\mathbb{U}$. Taking the logarithmic differentiation in (3.8) and using identity (1.6), we deduce that

$$
\begin{aligned}
& \operatorname{Re}\left((1-\alpha) \frac{I_{p}^{n}(\lambda, \mu) f(z)}{I_{p}^{n+1}(\lambda, \mu) f(z)}+\alpha \frac{I_{p}^{n-1}(\lambda, \mu) f(z)}{I_{p}^{n}(\lambda, \mu) f(z)}\right)-\frac{\eta}{p} \\
& \quad \geqq\left(1-\frac{\eta}{p}\right)\left[\operatorname{Re}(u(z))-\frac{p \alpha\left|z u^{\prime}(z)\right|}{(p+\lambda)(|\eta+(p-\eta) u(z)|)}\right] .
\end{aligned}
$$

Now, by using the well-known [16] estimates

$$
\left|z u^{\prime}(z)\right| \leqq \frac{2 r}{1-r^{2}} \operatorname{Re}(u(z)) \quad \text { and } \quad \operatorname{Re}(u(z)) \geqq \frac{1-r}{1+r} \quad(|z|=r<1)
$$

in (3.9), we obtain

$$
\begin{aligned}
& \operatorname{Re}\left((1-\alpha) \frac{I_{p}^{n}(\lambda, \mu) f(z)}{I_{p}^{n+1}(\lambda, \mu) f(z)}+\alpha \frac{I_{p}^{n-1}(\lambda, \mu) f(z)}{I_{p}^{n}(\lambda, \mu) f(z)}\right)-\frac{\eta}{p} \\
& \quad \geqq\left(1-\frac{\eta}{p}\right) \operatorname{Re}(u(z))\left[1-\frac{2 p \alpha r}{(p+\lambda)\left(\eta\left(1-r^{2}\right)+(p-\eta)(1-r)^{2}\right)}\right],
\end{aligned}
$$

which is certainly positive if $r<R$, where $R$ is given by (3.7). 
To show that the bound $R$ is best possible, we consider the function $f \in \mathcal{A}_{p}$ defined by

$$
\frac{I_{p}^{n}(\lambda, \mu) f(z)}{I_{p}^{n+1}(\lambda, \mu) f(z)}=\frac{\eta}{p}+\left(1-\frac{\eta}{p}\right) \frac{1+z}{1-z} \quad(z \in \mathbb{U})
$$

Noting that

$$
\begin{aligned}
(1 & -\alpha) \frac{I_{p}^{n}(\lambda, \mu) f(z)}{I_{p}^{n+1}(\lambda, \mu) f(z)}+\alpha \frac{I_{p}^{n-1}(\lambda, \mu) f(z)}{I_{p}^{n}(\lambda, \mu) f(z)}-\frac{\eta}{p} \\
& =\left(1-\frac{\eta}{p}\right) \frac{1+z}{1-z}\left[1+\frac{2 p \alpha z}{(p+\lambda)\left(\eta\left(1-z^{2}\right)+(p-\eta)(1-z)^{2}\right)}\right]=0
\end{aligned}
$$

for $z=-R$, we complete the proof of Theorem 2 .

Remark For $n=-1, \lambda=0, \mu=1-p$ and $\alpha=1$, Theorem 2 yields the corresponding result contained in [15].

For a function $f \in \mathcal{A}_{p}$, the generalized Bernardi-Libera-Livingston integral operator $\mathcal{F}_{\delta, p}: \mathcal{A}_{p} \longrightarrow \mathcal{A}_{p}$ is defined by (cf., e.g., [17])

$$
\begin{aligned}
\mathcal{F}_{\delta, p}(f)(z) & =\frac{\delta+p}{z^{\delta}} \int_{0}^{z} t^{\delta-1} f(t) d t=\left(z^{p}+\sum_{k=1}^{\infty} \frac{\delta+p}{\delta+p+k} z^{p+k}\right) * f(z) \\
& =z^{p}{ }_{2} F_{1}(1, \delta+p ; \delta+p+1 ; z) * f(z) \quad(\delta>-p ; z \in \mathbb{U}) .
\end{aligned}
$$

For convenience, we write $\mathcal{F}_{\delta, p}(f)(z)=\mathcal{F}_{\delta, p}(z), z \in \mathbb{U}$. It readily follows from (3.10) that $f \in \mathcal{A}_{p} \Rightarrow \mathcal{F}_{\delta, p} \in \mathcal{A}_{p}$.

Theorem 3 Let $\delta$ be a real number satisfying

$$
(\delta-\lambda)(1-B)+(p+\lambda)(1-A) \geqq 0 .
$$

(i) Iff $\in \mathcal{S}_{p, \lambda, \mu}^{n}(A, B)$, then

$$
\frac{I_{p}^{n}(\lambda, \mu) \mathcal{F}_{\delta, p}(z)}{I_{p}^{n+1}(\lambda, \mu) \mathcal{F}_{\delta, p}(z)} \prec q(z)=\frac{1}{p+\lambda}\left(\frac{1}{Q(z)}-\delta+\lambda\right) \prec \frac{1+A z}{1+B z} \quad(z \in \mathbb{U})
$$

where

$$
Q(z)= \begin{cases}\int_{0}^{1} t^{\delta+p-1}\left(\frac{1+B t z}{1+B z}\right)^{(p+\lambda)(B-A) / B} d t & (B \neq 0) \\ \int_{0}^{1} t^{\delta+p-1} \exp \{(p+\lambda) A(t-1) z\} d t & (B=0)\end{cases}
$$

and $q$ is the best dominant of (3.12). Consequently, the operator $\mathcal{F}_{\delta, p}$ maps the class $\mathcal{S}_{p, \lambda, \mu}^{n}(A, B)$ into itself.

(ii) If $-1 \leqq B<0$ and $\delta \geqq \max \left\{-\frac{(p+\lambda)(1-A)}{1-B}+\lambda, \frac{(p+\lambda)(B-A)}{B}-p-1\right\}$, then

$$
f \in \mathcal{S}_{p, \lambda, \mu}^{n}(A, B) \quad \Longrightarrow \quad \mathcal{F}_{\delta, p} \in \mathcal{S}_{p, \lambda, \mu}^{n}(1-2 \tau,-1)
$$


where

$$
\tau=\frac{1}{p+\lambda}\left[(\delta+p)\left({ }_{2} F_{1}\left(1, \frac{(p+\lambda)(B-A)}{B} ; \delta+p+1 ; \frac{B}{B-1}\right)\right)^{-1}-\delta+\lambda\right] .
$$

The bound $\tau$ is best possible.

Proof From (1.5) and (3.10), it follows that

$$
z\left(I_{p}^{n+1}(\lambda, \mu) \mathcal{F}_{\delta, p}\right)^{\prime}(z)=(\delta+p) I_{p}^{n+1}(\lambda, \mu) f(z)-\delta I_{p}^{n+1}(\lambda, \mu) \mathcal{F}_{\delta, p}(z) \quad(z \in \mathbb{U}) .
$$

We put

$$
g(z)=z\left(\frac{I_{p}^{n+1}(\lambda, \mu) \mathcal{F}_{\delta, p}(z)}{z^{p}}\right)^{1 /(p+\lambda)}
$$

and $r_{1}=\sup \{r: g(z) \neq 0,0<|z|<r<1\}$. Choosing the principal branch of $g$, it follows that $g$ is a single-valued and is analytic in $\mathbb{U}_{r_{1}}$. Taking the logarithmic differentiation in (3.15) and using identity (1.6) for $\mathcal{F}_{\delta, p}$, we deduce that the function

$$
\varphi(z)=\frac{z g^{\prime}(z)}{g(z)}=\frac{I_{p}^{n}(\lambda, \mu) \mathcal{F}_{\delta, p}(z)}{I_{p}^{n+1}(\lambda, \mu) \mathcal{F}_{\delta, p}(z)}
$$

is analytic in $\mathbb{U}_{r_{1}}$ and $\varphi(0)=1$. Using identity (3.14) in (3.16), we obtain

$$
(\delta+p) \frac{I_{p}^{n+1}(\lambda, \mu) f(z)}{I_{p}^{n+1}(\lambda, \mu) \mathcal{F}_{\delta, p}(z)}=(p+\lambda) \frac{I_{p}^{n}(\lambda, \mu) \mathcal{F}_{\delta, p}(z)}{I_{p}^{n+1}(\lambda, \mu) \mathcal{F}_{\delta, p}(z)}+(\delta-p) \quad\left(z \in \mathbb{U}_{r_{1}}\right)
$$

Since $f \in \mathcal{S}_{p, \lambda, \mu}^{n}(A, B)$, it is clear that $I_{p}^{n+1}(\lambda, \mu) f(z) \neq 0$ in $0<|z|<1$. So, by (3.17), we get

$$
\frac{I_{p}^{n+1}(\lambda, \mu) \mathcal{F}_{\delta, p}(z)}{I_{p}^{n+1}(\lambda, \mu) f(z)}=\frac{\delta+p}{(p+\lambda) \varphi(z)+(\delta-\lambda)} \quad\left(z \in \mathbb{U}_{r_{1}}\right)
$$

Again, by taking the logarithmic differentiation in (3.18) followed by the use of identity (1.6) in the resulting equation, we get

$$
\frac{I_{p}^{n}(\lambda, \mu) f(z)}{I_{p}^{n+1}(\lambda, \mu) f(z)}=\varphi(z)+\frac{z \varphi^{\prime}(z)}{(p+\lambda) \varphi(z)+(\delta-\lambda)} \prec \frac{1+A z}{1+B z} \quad\left(z \in \mathbb{U}_{r_{1}}\right) .
$$

The proof of the remaining part is the same as that of [13, Theorem 1], and we choose to omit the details. The result is best possible as $q$ is the best dominant of (3.12).

Remark Letting $n=-1, \lambda=0, \mu=1-p, A=1-(2 \eta / p)(0 \leqq \eta<p)$ and $B=-1$ in Theorem 3 , we have the following implications [15, Corollary 3.4 and Remark 3.2]:

$$
\mathcal{F}_{\delta, p}\left(\mathcal{S}_{p}^{*}(\eta)\right) \subset \mathcal{S}_{p}^{*}(\sigma) \text { and } \quad \mathcal{F}_{\delta, p}\left(\mathcal{K}_{p}(\eta)\right) \subset \mathcal{K}_{p}(\sigma)
$$

where $\delta \geqq \max \{-\eta, p-2 \eta-1\}$ and $\sigma=(\delta+p)\left({ }_{2} F_{1}(1,2(p-\eta) ; \delta+p+1 ; 1 / 2)\right)^{-1}-\delta$. The containment relations are best possible, and they improve the corresponding work in [18] for suitable values of the parameters $p, \eta$ and $\delta$. 


\section{Properties involving the operator $I_{p}^{n}(\lambda, \mu)$}

Theorem 4 If $f \in \mathcal{A}_{p}$ satisfies

$$
(1-\alpha) \frac{I_{p}^{n+1}(\lambda, \mu) f(z)}{z^{p}}+\alpha \frac{I_{p}^{n}(\lambda, \mu) f(z)}{z^{p}} \prec \frac{1+A z}{1+B z} \quad(z \in \mathbb{U}),
$$

then

$$
\operatorname{Re}\left(\frac{I_{p}^{n+1}(\lambda, \mu) f(z)}{z^{p}}\right)>\varrho \quad(z \in \mathbb{U})
$$

where

$$
\varrho= \begin{cases}\frac{A}{B}+\left(1-\frac{A}{B}\right)(1-B)^{-1}{ }_{2} F_{1}\left(1,1 ; \frac{p+\lambda}{\alpha}+1 ; \frac{B}{B-1}\right) & (B \neq 0), \\ 1-\frac{(p+\lambda) A}{p+\lambda+\alpha} & (B=0) .\end{cases}
$$

The result is best possible.

Proof Setting

$$
\varphi(z)=\frac{I_{p}^{n+1}(\lambda, \mu) f(z)}{z^{p}} \quad(z \in \mathbb{U})
$$

we note that $\varphi$ is of the form (1.2) and is analytic in $\mathbb{U}$. On differentiating (4.3) and using identity (1.6) in the resulting equation, we deduce that

$$
(1-\alpha) \frac{I_{p}^{n+1}(\lambda, \mu) f(z)}{z^{p}}+\alpha \frac{I_{p}^{n}(\lambda, \mu) f(z)}{z^{p}}=\varphi(z)+\frac{\alpha}{p+\lambda} z \varphi^{\prime}(z) \prec \frac{1+A z}{1+B z} \quad(z \in \mathbb{U}) .
$$

The proof of the remaining part of the theorem follows by using Lemma 1 and the techniques that proved Theorem 4 in [13].

With a view to stating a well-known result, we denote by $\mathcal{P}(\gamma)$ the class of functions $\varphi$ of the form (1.2) which are analytic in $\mathbb{U}$ and satisfy the inequality

$$
\operatorname{Re}(\varphi(z))>\gamma \quad(0 \leqq \gamma<1 ; z \in \mathbb{U}) .
$$

It is known [19] that if $\varphi_{j} \in \mathcal{P}\left(\gamma_{j}\right)\left(0 \leqq \gamma_{j}<1 ; j=1,2\right)$, then

$$
\left(\varphi_{1} * \varphi_{2}\right) \in \mathcal{P}\left(\gamma_{3}\right),
$$

where $\gamma_{3}=1-2\left(1-\gamma_{1}\right)\left(1-\gamma_{2}\right)$. The bound $\gamma_{3}$ is best possible.

Theorem 5 If the functions $I_{p}^{n}(\lambda, \mu) f_{j} / z^{p} \in \mathcal{P}\left(A_{j}, B_{j}\right)\left(-1 \leqq B_{j}<A_{j} \leqq 1, f_{j} \in \mathcal{A}_{p} ; j=1,2\right)$, then the function $\mathfrak{h}$ defined in $\mathbb{U}$ by

$$
\mathfrak{h}(z)=I_{p}^{n+1}(\lambda, \mu)\left(f_{1} * f_{2}\right)(z)
$$

satisfies

$$
\operatorname{Re}\left(\frac{I_{p}^{n}(\lambda, \mu) \mathfrak{h}(z)}{I_{p}^{n+1}(\lambda, \mu) \mathfrak{h}(z)}\right)>0 \quad(z \in \mathbb{U})
$$


provided

$$
\frac{\left(A_{1}-B_{1}\right)\left(A_{2}-B_{2}\right)}{\left(1-B_{1}\right)\left(1-B_{2}\right)}<\frac{2(p+\lambda)+1}{2\left[\left({ }_{2} F_{1}(1,1 ; p+\lambda+1 ; 1 / 2)-2\right)^{2}+2(p+\lambda)\right]} .
$$

Proof We have

$$
\frac{I_{p}^{n}(\lambda, \mu) f_{j}}{z^{p}} \in \mathcal{P}\left(\gamma_{j}\right) \quad\left(\gamma_{j}=\frac{1-A_{j}}{1-B_{j}} ; j=1,2\right) .
$$

Hence, by using (4.5), we deduce that

$$
\begin{aligned}
& \operatorname{Re}\left(\frac{I_{p}^{n}(\lambda, \mu) \mathfrak{h}(z)}{z^{p}}+\frac{z}{p+\lambda}\left(\frac{I_{p}^{n}(\lambda, \mu) \mathfrak{h}(z)}{z^{p}}\right)^{\prime}\right) \\
& \quad=\operatorname{Re}\left(\frac{I_{p}^{n}(\lambda, \mu) f_{1}(z)}{z^{p}} * \frac{I_{p}^{n}(\lambda, \mu) f_{2}(z)}{z^{p}}\right)>1-2 \frac{\left(A_{1}-B_{1}\right)\left(A_{2}-B_{2}\right)}{\left(1-B_{1}\right)\left(1-B_{2}\right)} \quad(z \in \mathbb{U}),
\end{aligned}
$$

which, in view of Lemma 1 for

$$
\kappa=p+\lambda, \quad A=-1+4 \frac{\left(A_{1}-B_{1}\right)\left(A_{2}-B_{2}\right)}{\left(1-B_{1}\right)\left(1-B_{2}\right)} \quad \text { and } \quad B=-1,
$$

yields

$$
\operatorname{Re}\left(\frac{I_{p}^{n}(\lambda, \mu) \mathfrak{h}(z)}{z^{p}}\right)>1+\frac{\left(A_{1}-B_{1}\right)\left(A_{2}-B_{2}\right)}{\left(1-B_{1}\right)\left(1-B_{2}\right)}\left[{ }_{2} F_{1}\left(1,1 ; p+\lambda+1 ; \frac{1}{2}\right)-2\right] \quad(z \in \mathbb{U}) .
$$

From (4.9), by using Theorem 4 for

$$
\alpha=1, \quad A=-1-4 \frac{\left(A_{1}-B_{1}\right)\left(A_{2}-B_{2}\right)}{\left(1-B_{1}\right)\left(1-B_{2}\right)}\left[{ }_{2} F_{1}\left(1,1 ; p+\lambda+1 ; \frac{1}{2}\right)-2\right] \text { and } B=-1 \text {, }
$$

we deduce that

$$
\operatorname{Re}(\theta(z))>1-2 \frac{\left(A_{1}-B_{1}\right)\left(A_{2}-B_{2}\right)}{\left(1-B_{1}\right)\left(1-B_{2}\right)}\left[{ }_{2} F_{1}\left(1,1 ; p+\lambda+1 ; \frac{1}{2}\right)-2\right]^{2} \quad(z \in \mathbb{U}),
$$

where $\theta(z)=I_{p}^{n}(\lambda, \mu) \mathfrak{h}(z) / z^{p}$. If we put

$$
\varphi(z)=\frac{I_{p}^{n}(\lambda, \mu) \mathfrak{h}(z)}{I_{p}^{n+1}(\lambda, \mu) \mathfrak{h}(z)} \quad(z \in \mathbb{U})
$$

then $\varphi$ is of the form (1.2) analytic in $\mathbb{U}$, and a simple computation shows that

$$
\begin{aligned}
\frac{I_{p}^{n}(\lambda, \mu) \mathfrak{h}(z)}{z^{p}}+\frac{z}{p+\lambda}\left(\frac{I_{p}^{n}(\lambda, \mu) \mathfrak{h}(z)}{z^{p}}\right)^{\prime} & =\theta(z)\left[(\varphi(z))^{2}+\frac{1}{p+\lambda} z \varphi^{\prime}(z)\right] \\
& =\Psi\left(\varphi(z), z \varphi^{\prime}(z) ; z\right),
\end{aligned}
$$

where $\Psi(u, v ; z)=\theta(z)\left(u^{2}+(v /(p+\lambda))\right)$. Thus, by using (4.8) in (4.11), we conclude that

$$
\operatorname{Re}\left(\Psi\left(\varphi(z), z \varphi^{\prime}(z) ; z\right)\right)>1-2 \frac{\left(A_{1}-B_{1}\right)\left(A_{2}-B_{2}\right)}{\left(1-B_{1}\right)\left(1-B_{2}\right)} \quad(z \in \mathbb{U}) .
$$


Now, for all real $x, y \leqq-\left(1+x^{2}\right) / 2$, we have

$$
\begin{aligned}
\operatorname{Re}(\Psi(i x, y ; z)) & =\left(\frac{y}{p+\lambda}-x^{2}\right) \operatorname{Re}(\theta(z)) \\
& \leqq-\frac{1}{2(p+\lambda)}\left[1+(2(p+\lambda)+1) x^{2}\right] \operatorname{Re}(\theta(z)) \\
& \leqq \frac{1}{2(p+\lambda)} \operatorname{Re}(\theta(z)) \leqq 1-2 \frac{\left(A_{1}-B_{1}\right)\left(A_{2}-B_{2}\right)}{\left(1-B_{1}\right)\left(1-B_{2}\right)} \quad(z \in \mathbb{U})
\end{aligned}
$$

by (4.7) and (4.10). Hence, by making use of Lemma 2, we get $\operatorname{Re}(\varphi(z))>0$ in $\mathbb{U}$. This completes the proof of Theorem 5 .

Theorem 6 If the functions $I_{p}^{n}(\lambda, \mu) f_{j} / z^{p} \in \mathcal{P}\left(A_{j}, B_{j}\right)\left(-1 \leqq B_{j}<A_{j} \leqq 1, f_{j} \in \mathcal{A}_{p} ; j=1,2,3\right)$, then the function $H$ defined in $\mathbb{U}$ by

$$
H(z)=I_{p}^{n+1}(\lambda, \mu)\left(f_{1} * f_{2} * f_{3}\right)(z)
$$

satisfies

$$
\operatorname{Re}\left(\frac{I_{p}^{2 n}(\lambda, \mu) H(z)}{I_{p}^{2 n+1}(\lambda, \mu) H(z)}\right)>0 \quad(z \in \mathbb{U})
$$

provided

$$
\frac{\left(A_{1}-B_{1}\right)\left(A_{2}-B_{2}\right)\left(A_{3}-B_{3}\right)}{\left(1-B_{1}\right)\left(1-B_{2}\right)\left(1-B_{3}\right)}<\frac{2(p+\lambda)+1}{4\left[\left({ }_{2} F_{1}(1,1 ; p+\lambda+1 ; 1 / 2)-2\right)^{2}+2(p+\lambda)\right]} .
$$

Proof From the definition of the function $H$, it is easily seen that

$$
\begin{aligned}
\operatorname{Re} & \left(\frac{I_{p}^{2 n}(\lambda, \mu) H(z)}{z^{p}}+\frac{z}{p+\lambda}\left(\frac{I_{p}^{2 n}(\lambda, \mu) H(z)}{z^{p}}\right)^{\prime}\right) \\
& =\operatorname{Re}\left(\frac{I_{p}^{n}(\lambda, \mu) f_{1}(z)}{z^{p}} * \frac{I_{p}^{n}(\lambda, \mu) f_{2}(z)}{z^{p}} * \frac{I_{p}^{n}(\lambda, \mu) f_{3}(z)}{z^{p}}\right) \\
& >1-4 \frac{\left(A_{1}-B_{1}\right)\left(A_{2}-B_{2}\right)\left(A_{3}-B_{3}\right)}{\left(1-B_{1}\right)\left(1-B_{2}\right)\left(1-B_{3}\right)} \quad(z \in \mathbb{U})
\end{aligned}
$$

and the proof of the theorem is completed similarly to Theorem 5 .

Theorem 7 Let $f_{j} \in \mathcal{A}_{p}(j=1,2)$. If the function $\mathfrak{h}$ defined in $\mathbb{U}$ by (4.6) satisfies

$$
\operatorname{Re}\left(\frac{I_{p}^{n}(\lambda, \mu) \mathfrak{h}(z)}{z^{p}}\right)>1-\frac{2(p+\lambda)+1}{2\left[\left({ }_{2} F_{1}(1,1 ; p+\lambda+1 ; 1 / 2)-2\right)^{2}+2(p+\lambda)\right]} \quad(z \in \mathbb{U}),
$$

then

$$
\operatorname{Re}\left(\frac{I_{p}^{n}(\lambda, \mu) \mathcal{G}(z)}{I_{p}^{n+1}(\lambda, \mu) \mathcal{G}(z)}\right)>0 \quad(z \in \mathbb{U})
$$


where

$$
\mathcal{G}(z)=\frac{p+\lambda}{z^{\lambda}} \int_{0}^{z} t^{\lambda-1} \mathfrak{h}(t) d t \quad(z \in \mathbb{U}) .
$$

Proof Using the fact that

$$
\begin{aligned}
\operatorname{Re}\left(\frac{I_{p}^{n}(\lambda, \mu) \mathfrak{h}(z)}{z^{p}}\right) & =\operatorname{Re}\left(\frac{I_{p}^{n}(\lambda, \mu) \mathcal{G}(z)}{z^{p}}+\frac{z}{p+\lambda}\left(\frac{I_{p}^{n}(\lambda, \mu) \mathcal{G}(z)}{z^{p}}\right)^{\prime}\right) \\
& >1-\frac{2(p+\lambda)+1}{2\left[\left({ }_{2} F_{1}(1,1 ; p+\lambda+1 ; 1 / 2)-2\right)^{2}+2(p+\lambda)\right]} \quad(z \in \mathbb{U})
\end{aligned}
$$

and by following the same lines of proof as in Theorem 5 , we get the required result.

Remark Putting $n=-1, \lambda=0$ and $\mu=1$ in Theorems 5, 6 and 7, respectively, we obtain the corresponding results contained in [20].

Theorem 8 Let $\delta>0$ and $0<\gamma \leqq(1+\delta(p+\lambda)) / \sqrt{1+2 \delta(p+\lambda)+2(\delta(p+\lambda))^{2}}$. If $f \in \mathcal{A}_{p}$ satisfies

$$
\frac{I_{p}^{n-1}(\lambda, \mu) f(z)}{z^{p}}\left(\frac{I_{p}^{n}(\lambda, \mu) f(z)}{z^{p}}\right)^{\delta-1} \prec 1+\gamma z \quad(z \in \mathbb{U})
$$

then $f \in \mathcal{S}_{p, \lambda, \mu}^{n-1}(1-2 \varkappa,-1)$, where

$$
\varkappa= \begin{cases}\frac{1+\delta(p+\lambda)}{1+\delta(p+\lambda)(1+\gamma)} & \left(0<\gamma \leqq \frac{1+\delta(p+\lambda)}{1+2 \delta(p+\lambda)}\right) \\ M_{p}(\lambda, \delta, \gamma) & \left(\frac{1+\delta(p+\lambda)}{1+2 \delta(p+\lambda)} \leqq \gamma \leqq \frac{1+\delta(p+\lambda)}{\sqrt{1+2 \delta(p+\lambda)+2(\delta(p+\lambda))^{2}}}\right)\end{cases}
$$

and

$$
M_{p}(\lambda, \delta, \gamma)=\frac{(1+\delta(p+\lambda))^{2}-\left[1+2 \delta(p+\lambda)+2(\delta(p+\lambda))^{2}\right] \gamma^{2}}{2\left[(1+\delta(p+\lambda))^{2}-(\delta(p+\lambda))^{2} \gamma^{2}\right]}
$$

Further, for $0<\gamma \leqq(1+\delta(p+\lambda)) /(1+3 \delta(p+\lambda))$,

$$
\left|\frac{I_{p}^{n-1}(\lambda, \mu) f(z)}{I_{p}^{n}(\lambda, \mu) f(z)}-1\right|<\frac{(1+2 \delta(p+\lambda)) \gamma}{1+\delta(p+\lambda)(1-\gamma)} \quad(z \in \mathbb{U}) .
$$

The bound given by (4.13) and the estimate in (4.14) are best possible.

Proof Setting

$$
\Theta(z)=\left(\frac{I_{p}^{n}(\lambda, \mu) f(z)}{z^{p}}\right)^{\delta} \quad(z \in \mathbb{U})
$$

and choosing the principal branch in (4.15), we note that $\Theta$ is analytic in $\mathbb{U}$ with $\Theta(0)=1$. A simple computation shows that (4.12) is equivalent to

$$
\Theta(z)+\frac{z \Theta^{\prime}(z)}{\delta(p+\lambda)} \prec 1+\gamma z \quad(z \in \mathbb{U}) .
$$


Now, by applying Lemma 1 (with $\kappa=\delta(p+\lambda), A=\gamma$ and $B=-1$ ), we get

$$
\Theta(z) \prec 1+\gamma_{1} z \quad\left(\gamma_{1}=\frac{\delta(p+\lambda) \gamma}{1+\delta(p+\lambda)} ; z \in \mathbb{U}\right)
$$

We further observe that

$$
1+\frac{1}{\delta(p+\lambda)} \frac{z \Theta^{\prime}(z)}{\Theta(z)}=\frac{I_{p}^{n-1}(\lambda, \mu) f(z)}{I_{p}^{n}(\lambda, \mu) f(z)} \quad(z \in \mathbb{U})
$$

Hence, assertion (4.13) follows by using part (i) of Lemma 3. If we put

$$
\frac{I_{p}^{n-1}(\lambda, \mu) f(z)}{I_{p}^{n}(\lambda, \mu) f(z)}=1+\omega(z) \quad(\omega \in \mathcal{B} ; z \in \mathbb{U})
$$

then we obtain (4.14) from part (ii) of Lemma 3.

To show that the estimates are best possible, we consider the function $f \in \mathcal{A}_{p}$ defined in U by

$$
\left(\frac{I_{p}^{n}(\lambda, \mu) f(z)}{z^{p}}\right)^{\delta}=1+\delta(p+\lambda) \gamma \int_{0}^{1} t^{\delta(p+\lambda)-1} \omega(t z) d t \quad(\delta>0, \omega \in \mathcal{B} ; z \in \mathbb{U}) .
$$

From this, we obtain

$$
\frac{I_{p}^{n-1}(\lambda, \mu) f(z)}{I_{p}^{n}(\lambda, \mu) f(z)}=\frac{1+\gamma \omega(z)}{1+\delta(p+\lambda) \gamma \int_{0}^{1} t^{\delta(p+\lambda)-1} \omega(t z) d t} \quad(z \in \mathbb{U})
$$

and the sharpness follows from Lemma 4 (for $\left.\delta^{\prime}=\delta(p+\lambda)\right)$.

Putting $n=\lambda=0, \mu=1-p$ and $\delta=1$ in Theorem 8 , we get the following.

Corollary 3 If $0<\gamma \leqq(p+1) / \sqrt{1+2 p+2 p^{2}}$ and $f \in \mathcal{A}_{p}$ satisfies

$$
\left|\frac{f^{\prime}(z)}{p z^{p-1}}-1\right|<\gamma \quad(z \in \mathbb{U})
$$

then

$$
\operatorname{Re}\left(\frac{z f^{\prime}(z)}{f(z)}\right)> \begin{cases}\frac{p(p+1)(1-\gamma)}{1+p(1+\gamma)} & \left(0<\gamma \leqq \frac{p+1}{2 p+1}\right) \\ \frac{p(p+1)^{2}-p\left(1+2 p+2 p^{2}\right) \gamma^{2}}{2\left((p+1)^{2}-(p \gamma)^{2}\right)} & \left(\frac{p+1}{2 p+1} \leqq \gamma \leqq \frac{p+1}{\sqrt{1+2 p+2 p^{2}}}\right)\end{cases}
$$

Further, for $0<\gamma \leqq(p+1) /(3 p+1)$,

$$
\left|\frac{z f^{\prime}(z)}{f(z)}-1\right|<\frac{p(2 p+1) \gamma}{1+p(1-\gamma)} \quad(z \in \mathbb{U})
$$

The estimates are best possible. 
Theorem 9 If $\gamma>0$ and $f \in \mathcal{A}_{p}$ satisfies

$$
(1-\alpha) \frac{I_{p}^{n}(\lambda, \mu) f(z)}{z^{p}}+\alpha \frac{I_{p}^{n-1}(\lambda, \mu) f(z)}{z^{p}} \prec 1+\gamma z \quad(\alpha>0 ; z \in \mathbb{U})
$$

then $f \in \mathcal{S}_{p, \lambda, \mu}^{n-1}(1-2 \vartheta,-1)$, where

$$
\vartheta= \begin{cases}\frac{\alpha((p+\lambda)(1+\gamma)+\alpha-\gamma)-2(p+\lambda) \gamma}{\alpha((p+\lambda)(1+\gamma)+\alpha)} & \left(0<\gamma \leqq \frac{p+\lambda+\alpha}{2(p+\lambda)+\alpha}\right), \\ \frac{N_{p}(\lambda, \alpha, \gamma)-(1-\alpha)}{\alpha} & \left(\frac{p+\lambda+\alpha}{2(p+\lambda)+\alpha} \leqq \gamma \leqq \frac{p+\lambda+\alpha}{\sqrt{(p+\lambda)^{2}+(p+\lambda+\alpha)^{2}}}\right)\end{cases}
$$

and

$$
N_{p}(\lambda, \alpha, \gamma)=\frac{(p+\lambda+\alpha)^{2}-\left((p+\lambda+\alpha)^{2}+(p+\lambda)^{2}\right) \gamma^{2}}{2\left((p+\lambda+\alpha)^{2}-(p+\lambda)^{2} \gamma^{2}\right)} \text {. }
$$

For $0<\gamma \leqq(p+\lambda+\alpha) /(3(p+\lambda)+\alpha)$, we have

$$
\left|\frac{I_{p}^{n-1}(\lambda, \mu) f(z)}{I_{p}^{n}(\lambda, \mu) f(z)}-1\right|<\frac{(2(p+\lambda)+\alpha) \gamma}{(p+\lambda)(1-\gamma)+\alpha} \quad(z \in \mathbb{U}) .
$$

Further, $f \in \mathcal{S}_{p, \lambda, \mu}^{n}(1-2 \tilde{\varkappa},-1)$, where $\tilde{\varkappa}$ is obtained from $\varkappa$ (given in (4.13)) for $\delta=1$ and upon replacing $\gamma$ by $(p+\lambda) \gamma /(p+\lambda+\alpha)$. Moreover, for $0<\gamma \leqq((p+\lambda+\alpha)(p+\lambda+1)) /$ $[(p+\lambda)(1+2(p+\lambda))]$

$$
\left|\frac{I_{p}^{n}(\lambda, \mu) f(z)}{I_{p}^{n+1}(\lambda, \mu) f(z)}-1\right|<\frac{(p+\lambda)(1+2(p+\lambda)) \gamma}{(p+\lambda+\alpha)+(p+\lambda)(\alpha+(p+\lambda)(1-\gamma))} \quad(z \in \mathbb{U}) .
$$

The estimates are best possible.

Proof Since $f \in \mathcal{A}_{p}$ satisfies (4.16), by Theorem 4 (for $A=\gamma$ and $B=-1$ ) we obtain

$$
\frac{I_{p}^{n}(\lambda, \mu) f(z)}{z^{p}} \prec 1+\gamma_{1} z \quad\left(\gamma_{1}=\frac{(p+\lambda) \gamma}{p+\lambda+\alpha} ; z \in \mathbb{U}\right)
$$

Again, on writing (4.16) in the form

$$
\frac{I_{p}^{n}(\lambda, \mu) f(z)}{z^{p}}\left(1-\alpha+\alpha \frac{I_{p}^{n-1}(\lambda, \mu) f(z)}{I_{p}^{n}(\lambda, \mu) f(z)}\right) \prec 1+\gamma z \quad(z \in \mathbb{U})
$$

and using part (ii) of Lemma 3, we deduce that

$$
\begin{aligned}
& \operatorname{Re}\left(1-\alpha+\alpha \frac{I_{p}^{n-1}(\lambda, \mu) f(z)}{I_{p}^{n}(\lambda, \mu) f(z)}\right) \\
& \quad \geqq \begin{cases}\frac{(p+\lambda+\alpha)(1-\gamma)}{(p+\lambda)(1+\gamma)+\alpha} & \left(0<\gamma \leqq \frac{p+\lambda+\alpha}{2(p+\lambda)+\alpha}\right), \\
N_{p}(\lambda, \alpha, \mu) & \left(\frac{p+\lambda+\alpha}{2(p+\lambda)+\alpha} \leqq \gamma \leqq \frac{p+\lambda+\alpha}{\sqrt{(p+\lambda)^{2}+(p+\lambda+\alpha)^{2}}}\right),\end{cases}
\end{aligned}
$$


which implies assertion (4.17). By using part (ii) of Lemma 3 with

$$
\omega(z)=\alpha\left(\frac{I_{p}^{n-1}(\lambda, \mu) f(z)}{I_{p}^{n}(\lambda, \mu) f(z)}-1\right)
$$

we obtain (4.18). That $f \in \mathcal{S}_{p, \lambda, \mu}^{n-1}(1-2 \tilde{\varkappa},-1)$ and (4.19) now follow from Theorem 8 and (4.20).

To show the sharpness of the estimates, we consider the function $f$ defined in $\mathbb{U}$ by

$$
\frac{I_{p}^{n}(\lambda, \mu) f(z)}{z^{p}}=1+\frac{(p+\lambda) \gamma}{\alpha} \int_{0}^{1} t^{\frac{p+\lambda}{\alpha}-1} \omega(t z) d t \quad(\omega \in \mathcal{B} ; z \in \mathbb{U}) .
$$

Hence, by using identity (1.6), we get

$$
1-\alpha+\alpha \frac{I_{p}^{n-1}(\lambda, \mu) f(z)}{I_{p}^{n}(\lambda, \mu) f(z)}=\frac{1+\gamma \omega(z)}{1+\frac{(p+\lambda) \gamma}{\alpha} \int_{0}^{1} t^{\frac{p+\lambda}{\alpha}-1} \omega(t z) d t} \quad(z \in \mathbb{U}),
$$

and the sharpness follows from Lemma 4 . The fact that $f \in \mathcal{S}_{p, \lambda, \mu}^{n}\left(1-2 \tilde{\varkappa}_{,}-1\right)$ is sharp follows from (4.20) and the sharpness of Theorem 8.

Putting $n=-1, \lambda=0$ and $\mu=1-p$ in Theorem 9, we have the following.

Corollary 4 If $\in \mathcal{A}_{p}$ satisfies

$$
\left(1-\alpha+\frac{\alpha}{p}\right) \frac{f^{\prime}(z)}{p z^{p-1}}+\alpha \frac{f^{\prime \prime}(z)}{p^{2} z^{p-2}} \prec 1+\gamma z \quad(\gamma>0, \alpha>0 ; z \in \mathbb{U}),
$$

then

$$
\operatorname{Re}\left(1+\frac{z f^{\prime \prime}(z)}{f^{\prime}(z)}\right)> \begin{cases}\frac{p \alpha(p+\alpha+(p-1) \gamma)-2 p^{2} \gamma}{\alpha(p(1+\gamma)+\alpha)} & \left(0<\gamma \leqq \frac{p+\alpha}{2 p+\alpha}\right), \\ \frac{p(p+\alpha)^{2}-p\left((p+\alpha)^{2}+p^{2}\right) \gamma^{2}}{2 \alpha\left((p+\alpha)^{2}-p^{2} \gamma^{2}\right)}-p\left(\frac{1-\alpha}{\alpha}\right) & \left(\frac{p+\alpha}{2 p+\alpha} \leqq \gamma \leqq \frac{p+\alpha}{\sqrt{p^{2}+(p+\alpha)^{2}}}\right)\end{cases}
$$

and for $0<\gamma \leqq(p+\alpha) /(3 p+\alpha)$,

$$
\left|1+\frac{z f^{\prime \prime}(z)}{f^{\prime}(z)}-p\right|<\frac{p(2 p+\alpha) \gamma}{\alpha(p(1-\gamma)+\alpha)} \quad(z \in \mathbb{U}) .
$$

The result is sharp.

Remark For $p=1$ in Corollary 3 and Corollary 4, we get the corresponding results obtained in [11].

Theorem 10 Iff $\in \mathcal{A}_{p}$ satisfies

$$
\begin{aligned}
& \left|\frac{I_{p}^{n}(\lambda, \mu) f(z)}{I_{p}^{n+1}(\lambda, \mu) f(z)}-1\right|^{\gamma}\left|\frac{I_{p}^{n-1}(\lambda, \mu) f(z)}{I_{p}^{n}(\lambda, \mu) f(z)}-1\right|^{\beta} \\
& \quad<\left(\frac{A-B}{1+|B|}\right)^{\gamma+\beta}\left(1+\frac{1}{(p+\lambda)(1+|A|)}\right)^{\beta} \quad(z \in \mathbb{U}),
\end{aligned}
$$

for some real numbers $\beta$ and $\gamma$ such that $\beta \geqq 0, \gamma \geqq 0, \beta+\gamma>0$, then $f \in \mathcal{S}_{p, \lambda, \mu}^{n}(A, B)$. 
Proof If we set

$$
\frac{I_{p}^{n}(\lambda, \mu) f(z)}{I_{p}^{n+1}(\lambda, \mu) f(z)}=\frac{1+A \omega(z)}{1+B \omega(z)} \quad(z \in \mathbb{U})
$$

then $\omega$ is analytic in $\mathbb{U}$. Differentiating (4.22) logarithmically and using identity (1.6) in the resulting equation, we get

$$
\frac{I_{p}^{n-1}(\lambda, \mu) f(z)}{I_{p}^{n}(\lambda, \mu) f(z)}=\frac{1+A \omega(z)}{1+B \omega(z)}+\frac{(A-B) z \omega^{\prime}(z)}{(p+\lambda)(1+A \omega(z))(1+B \omega(z))} \quad(z \in \mathbb{U}) .
$$

Now, we have

$$
\begin{aligned}
& \left|\frac{I_{p}^{n}(\lambda, \mu) f(z)}{I_{p}^{n+1}(\lambda, \mu) f(z)}-1\right|^{\gamma}\left|\frac{I_{p}^{n-1}(\lambda, \mu) f(z)}{I_{p}^{n}(\lambda, \mu) f(z)}-1\right|^{\beta} \\
& =(A-B)^{\gamma+\beta}\left|\frac{\omega(z)}{1+B \omega(z)}\right|^{\gamma+\beta} \\
& \quad \times\left|1+\frac{1}{p+\lambda} \frac{z \omega^{\prime}(z)}{\omega(z)} \frac{1}{1+A \omega(z)}\right|^{\beta} \quad(z \in \mathbb{U}) .
\end{aligned}
$$

We claim that $|\omega(z)|<1$ for $z \in \mathbb{U}$. Otherwise, there exists a point $z_{0} \in \mathbb{U}$ such that $\max _{|z| \leqq\left|z_{0}\right|}|\omega(z)|=\left|\omega\left(z_{0}\right)\right|=1$. By using Jack's lemma [21], we write $\omega\left(z_{0}\right)=e^{i \theta}, 0<\theta \leqq 2 \pi$ and $z \omega^{\prime}\left(z_{0}\right)=m \omega\left(z_{0}\right), m \geqq 1$. Thus, from (4.23), it follows that

$$
\begin{aligned}
\left|\frac{I_{p}^{n}(\lambda, \mu) f\left(z_{0}\right)}{I_{p}^{n+1}(\lambda, \mu) f\left(z_{0}\right)}-1\right|^{\gamma}\left|\frac{I_{p}^{n-1}(\lambda, \mu) f\left(z_{0}\right)}{I_{p}^{n}(\lambda, \mu) f\left(z_{0}\right)}-1\right|^{\beta} & \geqq\left(\frac{A-B}{1+|B|}\right)^{\gamma+\beta}\left(1+\frac{m}{p+\lambda} \Re\left(\frac{1}{1+A e^{i \theta}}\right)\right)^{\beta} \\
& \geqq\left(\frac{A-B}{1+|B|}\right)^{\gamma+\beta}\left(1+\frac{1}{(p+\lambda)(1+|A|)}\right)^{\beta} .
\end{aligned}
$$

This contradicts the hypothesis (4.21) and hence $|\omega(z)|<1$ for $z \in \mathbb{U}$. This proves the theorem.

Taking $A=1-\frac{2 \rho}{p}, B=-1, \mu=1-p, \lambda=0, n=-1$ and $\gamma=1-\beta$ in Theorem 10 , we get the following interesting criterion for starlikeness for multivalent functions, which improves the corresponding work in [22] for $p=1$.

Corollary 5 Let $\beta \geqq 0$ and $0 \leqq \rho<p$. Iff $\in \mathcal{A}_{p}$ satisfies

$$
\left|\frac{z f^{\prime}(z)}{f(z)}-p\right|^{1-\beta}\left|1+\frac{z f^{\prime \prime}(z)}{f^{\prime}(z)}-p\right|^{\beta}<\xi(p, \rho, \beta)= \begin{cases}(p-\rho)\left(1+\frac{1}{2(p-\rho)}\right)^{\beta} & \left(0 \leqq \rho \leqq \frac{p}{2}\right) \\ (p-\rho)\left(1+\frac{1}{2 \rho}\right)^{\beta} & \left(\frac{p}{2} \leqq \rho<p\right)\end{cases}
$$

for all $z \in \mathbb{U}$, then $f \in \mathcal{S}_{p}^{*}(\rho)$.

Similarly, by setting $A=1-\frac{2 \rho}{p}, B=-1, \mu=1-p, \lambda=0, n=-2$ and $\gamma=1-\beta$ in Theorem 10 , we obtain the following sufficient condition for convexity of multivalent functions. 
Corollary 6 Let $\beta \geqq 0$ and $0 \leqq \rho<p$.Iff $\in \mathcal{A}_{p}$ satisfies

$$
\left|\left(1+\frac{z f^{\prime \prime}(z)}{f^{\prime}(z)}\right)-p\right|^{1-\beta}\left|\left(1+\frac{z^{2} f^{\prime \prime \prime}(z)+2 z f^{\prime \prime}(z)}{z f^{\prime \prime}(z)+f^{\prime}(z)}\right)-p\right|^{\beta}<\xi(p, \rho, \beta) \quad(z \in \mathbb{U})
$$

where $\xi(p, \rho, \beta)$ is defined as in Corollary 5 , then $f \in \mathcal{K}_{p}(\rho)$.

\section{Competing interests}

The authors declare that they have no competing interests.

\section{Authors' contributions}

All authors jointly worked on the results and they read and approved the final manuscript.

\section{Author details}

${ }^{1}$ Department of Mathematics, Utkal University, Vani Vihar, Bhubaneswar, 751004, India. ${ }^{2}$ Department of Applied Mathematics, Pukyong National University, Busan, Korea. ${ }^{3}$ Department of Mathematics, Bhadrak Institute of Engineering and Technology, Bhadrak, 756 113, India.

\section{Acknowledgements}

This work was supported by a Research Grant of Pukyong National University (2013 year) and the Basic Science Research Program through the National Research Foundation of Korea (NRF) funded by the Ministry of Education, Science and Technology (No. 2011-0007037)

Received: 3 January 2013 Accepted: 1 August 2013 Published: 21 September 2013

\section{References}

1. Janowski, W: Some extremal problems for certain families of analytic functions I. Ann. Pol. Math. 28, $297-326$ (1973)

2. Whittaker, ET, Watson, GN: A Course on Modern Analysis, 4th edn. (reprinted) Cambridge University Press, Cambridge (1927)

3. Cho, NE, Srivastava, HM: Argument estimates of certain analytic functions defined by a class of multiplier transformations. Math. Comput. Model. 37, 39-49 (2003)

4. Flett, TM: The dual of an inequality of Hardy and Littlewood and some related inequalities. J. Math. Anal. Appl. 38, 746-765 (1972)

5. Sălăgean, GS: Subclasses of univalent functions. In: Complex Analysis - Fifth Romanian-Finnish Seminar, Part I, Bucharest, 1981. Lecture Notes in Math., vol. 1013, pp. 362-372. Springer, Berlin (1983)

6. Cho, NE, Kim, TH: Multiplier transformations and strongly close-to-convex functions. Bull. Korean Math. Soc. 40, 399-410 (2003)

7. Cho, NE, Kim, JA: Inclusion properties of certain subclasses of analytic functions defined by a multiplier transformation. Comput. Math. Appl. 52, 323-330 (2006)

8. Uralegaddi, BA, Somanatha, C: Certain classes of univalent functions. In: Current Topics in Analytic Function Theory, pp. 371-374. World Scientific, River Edge (1992)

9. Hallenbeck, DJ, Ruscheweyh, S: Subordination by convex functions. Proc. Am. Math. Soc. 52, 191-195 (1975)

10. Miller, SS, Mocanu, PT: Differential Subordinations, Theory and Applications. Dekker, New York (2000)

11. Ponnusamy, S, Singh, V: Convolution properties of some classes of analytic functions. Internal Report, SPIC Science Foundation (1990)

12. Miller, SS, Mocanu, PT: Univalent solutions of Briot-Bouquet differential subordinations. J. Differ. Equ. 56, 297-309 (1985)

13. Patel, J, Cho, NE, Srivastava, HM: Certain classes of multivalent functions associated with a family of linear operators. Math. Comput. Model. 43, 320-338 (2006)

14. Miller, SS, Mocanu, PT, Reade, MO: The order of starlikeness of $\alpha$-convex functions. Mathematica 20(43), 25-30 (1978)

15. Patel, J, Cho, NE: Some classes of analytic functions involving Noor integral operator. J. Math. Anal. Appl. 312, 564-575 (2005)

16. MacGregor, TH: Subordination of convex functions of order $\alpha$. J. Lond. Math. Soc. 9(2), 530-536 (1975)

17. Choi, JH, Saigo, M, Srivastava, HM: Some inclusion properties of a certain family of integral operators. J. Math. Anal. Appl. 276, 432-445 (2002)

18. Fukui, S, Kim, JA, Srivastava, HM: On certain subclasses of univalent functions by some integral operators. Math. Jpn. 50, 359-370 (1999)

19. Stankiewicz, J, Stankiewicz, Z: Some applications of the Hadamard convolution in the theory of functions. Ann. Univ. Mariae Curie-SkTodowska, Sect. A 40, 251-256 (1986)

20. Lashin, AY: Some convolution properties of analytic functions. Appl. Math. Lett. 18, 135-138 (2005)

21. Jack, IS: Functions starlike and convex of order $\alpha$. J. Lond. Math. Soc. 3(2), 469-474 (1971)

22. Owa, S, Srivastava, HM: Univalent and starlike generalized hypergeometric functions. Can. J. Math. 39, 1057-1077 (1987) 